University of Wollongong

Research Online

Faculty of Engineering - Papers (Archive)

Faculty of Engineering and Information

Sciences

April 2006

\title{
Transport of spin-polarized electrons in a magnetic superlattice
}

J. Liu

Peking University, Beijing, China

W. Deng

South China University of Science and Technology, China

K. Xia

Chinese Academy of Sciences, China

C. Zhang

University of Wollongong, czhang@uow.edu.au

Z. Ma

Peking University, China

Follow this and additional works at: https://ro.uow.edu.au/engpapers

Part of the Engineering Commons

https://ro.uow.edu.au/engpapers/219

\section{Recommended Citation}

Liu, J.; Deng, W.; Xia, K.; Zhang, C.; and Ma, Z.: Transport of spin-polarized electrons in a magnetic superlattice 2006.

https://ro.uow.edu.au/engpapers/219

Research Online is the open access institutional repository for the University of Wollongong. For further information contact the UOW Library: research-pubs@uow.edu.au 


\title{
Transport of spin-polarized electrons in a magnetic superlattice
}

\author{
Jun-Feng Liu, ${ }^{1,2}$ Wen-Ji Deng, ${ }^{3}$ Ke Xia, ${ }^{4}$ Chao Zhang, ${ }^{5}$ and Zhongshui Ma ${ }^{1,5}$ \\ ${ }^{1}$ State Key Laboratory for Mesoscopic Physics and Department of Physics, Peking University, Beijing 100871, China \\ ${ }^{2}$ Department of Physics, Zhongshan University, Guangzhou 510275, China \\ ${ }^{3}$ Department of Applied Physics, South China University of Science and Technology, Guangzhou 510641, People's Republic of China \\ ${ }^{4}$ Institute of Physics, Chinese Academy of Sciences, Beijing 100080, China \\ ${ }^{5}$ School of Engineering Physics, University of Wollongong, New South Wales 2522, Australia \\ (Received 1 November 2005; revised manuscript received 12 January 2006; published 10 April 2006)
}

\begin{abstract}
We investigated the ballistic transport of two-dimensional electrons subjected to a periodically modulated magnetic field in the presence of the spin-orbit coupling of both the Rashba and the Dresselhaus types. It is shown that the spin splitting leads to additional gaps in the band structure and a series of minima in the transmission probability. The boundaries of the superlattice cause a finite spin polarization which can be tuned by the magnetic field, the electronic energy, and the superlattice period. The potential of such magnetic modulated structures as spin filters is discussed.
\end{abstract}

DOI: 10.1103/PhysRevB.73.155309

PACS number(s): 73.23.-b, 73.40.Gk

\section{INTRODUCTION}

The mean free path of electrons in two dimensional electron gases (2DEG) in semiconductors, such as a $\mathrm{GaAs} / \mathrm{Al}_{x} \mathrm{Ga}_{1-x} \mathrm{As}$ heterointerface, can be as long as several microns. This makes it possible to study the ballistic transport under potential modulation with a feature size of $100 \mathrm{~nm}$. In addition to the electric potential modulation, ${ }^{1}$ the magnetic field modulation has attracted vast attention. ${ }^{2}$ The experiment realization of nanometer scale magnetic field modulation can be achieved by patterning ferromagnetic ${ }^{3}$ or superconducting materials. ${ }^{4}$ The big advantage of using a magnetic field as potential modulation is that it can be done in a flexible fashion. ${ }^{5}$

The early theory and experiment ${ }^{3,4}$ were devoted to the interesting magnetoresistance oscillation as the function of low applied magnetic field, known as analog of the Weiss oscillation. ${ }^{1}$ Recently the theoretical development has focused on the spin dependent electronic transport properties of 2DEGs under magnetic modulations. ${ }^{6,7} \mathrm{~V}$. Kubrak et al. reported the measurement of the magnetoresistance due to a single magnetic barrier induced by a submicron ferromagnetic line. ${ }^{8}$

The prerequisite of the spintronics is to generate spin polarized electrons in high mobility semiconductors. ${ }^{9}$ The initial proposal of injecting spin from ferromagnetic metal into semiconductor by Datta and Das ${ }^{10}$ seems very difficult to be realized experimentally. Spin-orbit interaction as an alternative way to generate spin electronically has attracted much attention and some exciting progress has been achieved very recently. ${ }^{11,12}$

However, one big advantage of the proposal by Datta and Das, ${ }^{10}$ i.e., the device is nonvolatile, seems also lost when we generate spin electrically. A magnetic modulation generated by ferromagnetic pattern ${ }^{13}$ can be used to solve this problem. In this paper, we studied the ballistic spin-dependent transport through a 2DEG system modulated by a periodic magnetic potential in the presence of spin-orbit. The magnetic potential considered here is chosen to be the magnetic Kronig-Penney superlattice (MKP), ${ }^{6,7}$ which is modeled by an alternating sequence of equally spaced $\delta$ functions along the axis (see Fig. 1). We have studied the effect of spin splitting on the band structure and spin-dependent transmission probability of MKP model in the presence of Rashba interaction. The study shows that the MKP model can reproduce most of the results of the well known electrostatic Kronig-Penney (EKP) model but the barrier height can be tuned by the direction of magnetization now. The spin filter effects will also be discussed in this paper.

This paper is organized as follows. Section II is devoted to explain our model and to obtain the wave function of the Hamiltonian. In Sec. III, we calculate band structure and the transmission through a $N$-periods superlattice. In Sec. IV, we compare the MKP superlattice with a real superlattice. In Sec. V, we discuss the spin filter effect of the system and our conclusions are given in Sec. VI.

(a)

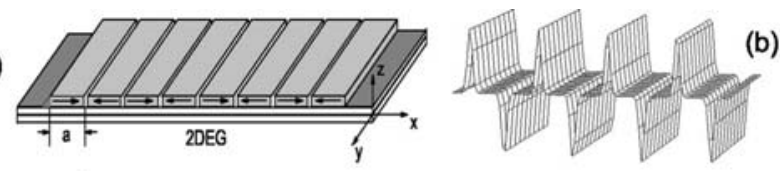

(c) $\underset{x}{x}$

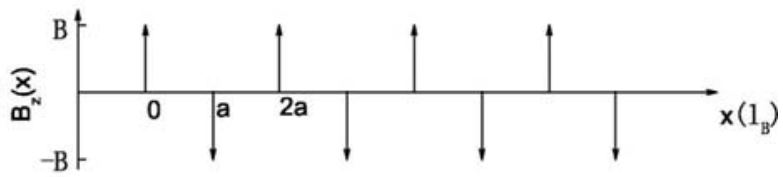

(d)

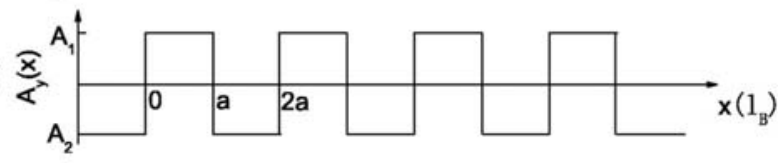

(e)

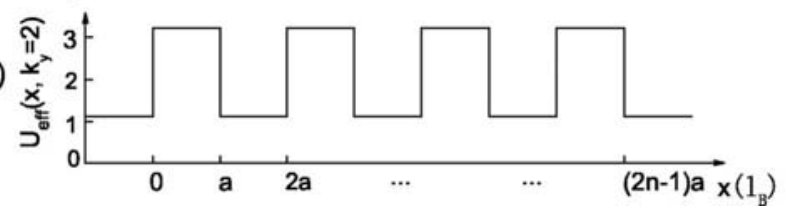

FIG. 1. (a) Schematic illustration of the device and (b) corresponding magnetic field. (c) The model magnetic field $B_{z}(x)$, (d) the vector potential $A_{y}(x)$, (e) the effective potential $U_{\text {eff }}\left(x, k_{y}=2\right)$ as functions of $x$. 


\section{MODEL AND METHOD}

We consider a 2DEG system with spin-orbit interaction and a static periodic magnetic field which is perpendicular to the plane of 2DEG. The spin-orbit interaction can be described in terms of two dominant contributions to the model Hamiltonian, the Rashba coupling stemming from the structure inversion asymmetry of confining potential, ${ }^{14}$ and the Dresselhaus coupling from the bulk inversion asymmetry. ${ }^{15}$ The magnetic field can be realized by patterning ferromagnetic strips with in-plane magnetization along the $x$ direction alternating in sign, as depicted in Fig. 1(a). The periodically modulated spikelike magnetic field is shown in Fig. 1(b) in 2DEG. The magnetic field can be simplified in the form of MKP model as illustrated in Fig. 1(c) so that the periodic magnetic field, which is perpendicular to the plane of 2DEG, takes the form of $\mathbf{B}(x)=B_{z}(x) \hat{e}_{z}$ with

$$
B_{z}(x)=B \sum_{n=-\infty}^{\infty}(-1)^{n} \delta(x-n a),
$$

where $a$ is the half period. In the Landau gauge, the vector potential is given as $\mathbf{A}(x)=\left[0, A_{y}(x), 0\right]$ with

$$
A_{y}(x)=\frac{B}{2} \operatorname{sgn}\left(\sin \frac{\pi x}{a}\right)
$$

In the effective-mass approximation, the Hamiltonian of an electron is given as

$$
\begin{aligned}
H= & \frac{1}{2 m^{*}}\left[\mathbf{p}+\frac{e}{c} \mathbf{A}\right]^{2}+g^{*} \frac{e \hbar}{4 m_{0} c^{2}} \sigma_{z} B_{z} \\
& +\left[\frac{\alpha(x)}{2 \hbar} \sigma \times\left(\mathbf{p}+\frac{e}{c} \mathbf{A}\right)+\left(\mathbf{p}+\frac{e}{c} \mathbf{A}\right) \times \sigma \frac{\alpha(x)}{2 \hbar}\right]_{z} \\
& +\frac{\beta(x)}{\hbar} \sigma_{y}\left[p_{y}+\frac{e}{c} A_{y}(x)\right]-\frac{1}{2 \hbar} \sigma_{x}\left[\beta(x) p_{x}+p_{x} \beta(x)\right],
\end{aligned}
$$

where $m^{*}$ is the effective mass of electron, $m_{0}$ is the freeelectron mass in vacuum, $g^{*}$ is the effective $g$ factor of the electron, and $\sigma=\left(\sigma_{x}, \sigma_{y}, \sigma_{z}\right)$ are the Pauli matrices. The second term in Eq. (3) represents the Zeeman splitting due to the local magnetic field. ${ }^{16}$ The third term is Rashba term with Rashba coupling coefficient $\alpha(x)$ which can be tuned by the gate voltage. ${ }^{17}$ It is written in the hermite form when Rashba coefficient depends on $x$, which is notable in the boundary conditions of interface between free electron region and Rashba coupling region. Similarly, the forth and fifth terms are Dresselhaus terms with the Dresselhaus coupling coefficient $\beta(x)$. In constructing the model, the possible straininduced electrostatic potentials modulation ${ }^{18}$ has been neglected.

Because of $\left[p_{y}, H\right]=0$, the system is translational invariant along the $y$ direction. So the wave function can be written as $\Psi(x, y)=e^{i k_{y} y} \psi(x)$, where $k_{y}$ is the wave vector in the $y$ direction. After substituting $\Psi$ into Schrödinger equation $H \Psi(x, y)=E \Psi(x, y)$ we obtain a one-dimensional (1D) equation for $\psi(x)$. In the rest of the paper, all the physical quantities are expressed in the dimensionless units: coordinate $\mathbf{x} \rightarrow \mathbf{x} l_{B}$, magnetic field $B_{z}(x) \rightarrow B_{z}(x) B_{0}$, vector potential $A_{y}(x) \rightarrow A_{y}(x) B_{0} l_{B}$, energy $E \rightarrow E_{0} E$ with $E_{0}=\hbar \omega_{c}$, and spinorbit coupling coefficient $\alpha \rightarrow \alpha E_{0} l_{B}, \beta \rightarrow \beta E_{0} l_{B} \cdot{ }^{19}$ Here $B_{0}$ is some characteristic magnetic field, $\omega_{c}=e B_{0} / m^{*} c$ is the cyclotron frequency and $l_{B}=\sqrt{\hbar c / e B_{0}}$ is the magnetic length. For InGaAs/InAlAs system ${ }^{17}$ and an estimated $B_{0}$ at $B_{0}=0.5 T$, we have $m^{*}=0.05 m_{0}, g^{*}=4, l_{B}=362.7 \AA$, and $E_{0}=\hbar \omega_{c}$ $=1.16 \mathrm{meV}$. The magnetic field is discontinuous only at $x$ $=n a$ and the Rashba and Dresselhaus coupling coefficients are discontinuous at two ends of the 2DEG. In each segment with constant vector potential $A_{y}$ and Rashba parameter $\alpha$ and Dresselhaus parameter $\beta$, the 1D Shrödinger equation for $\psi(x)$ is written as

$$
\begin{gathered}
\left\{\frac{1}{2} \frac{d^{2}}{d x^{2}}-U_{\mathrm{eff}}\left(x, k_{y}\right)-\alpha\left[K\left(x, k_{y}\right) \sigma_{x}+i \sigma_{y} \frac{d}{d x}\right]\right. \\
\left.-\beta\left[K\left(x, k_{y}\right) \sigma_{y}+i \sigma_{x} \frac{d}{d x}\right]+E\right\} \psi=0,
\end{gathered}
$$

where $K\left(x, k_{y}\right)=k_{y}+A_{y}(x), U_{e f f}\left(x, k_{y}\right)=K^{2}\left(x, k_{y}\right) / 2$ is the effective potential which is depicted in Fig. 1(e) for $k_{y}=2$. The important difference from the EKP model is that the barrier height of effective potential depends strongly on the transverse wave vector $k_{y}$.

The solution of Eq. (4) can be denoted as $\psi(x)=\xi e^{i k x}$, where $\xi$ is the two components spinor. We can find four eigenfunctions of Eq. (4): $\xi_{1} e^{i k_{1} x}, \xi_{2} e^{i k_{2} x}, \xi_{3} e^{i k_{3} x}$, and $\xi_{4} e^{i k_{4} x}$, with $\xi_{1}=\left(\begin{array}{c}1 \\ u_{1}\end{array}\right), \quad \xi_{2}=\left(\begin{array}{c}1 \\ u_{2}\end{array}\right), \xi_{3}=\left(\begin{array}{c}1 \\ u_{3}\end{array}\right)$, and $\xi_{4}=\left(\begin{array}{c}1 \\ u_{4}\end{array}\right)$. When both Rashba term and Dresselhaus term are considered, the symmetry between $k_{x}$ and $-k_{x}$ is broken. In general, the wave function in each segment can be expressed in terms of the linear combination of these four possible eigenfunctions, i.e.,

$$
\psi(x)=a \xi_{1} e^{i k_{1} x}+b \xi_{2} e^{i k_{2} x}+c \xi_{3} e^{i k_{3} x}+d \xi_{4} e^{i k_{4} x},
$$

where $a, b, c$, and $d$ are four coefficients, which should be determined by the boundary conditions. Because of the periodic structure of effective potential, the wave function satisfies the Bloch theorem, i.e., $\psi(x)=e^{i k \cdot 2 a} \psi(x-2 a)$, where $k$ is Bloch wave vector along the $x$ direction. To obtain the solution for the whole system, the wave function in every segment should satisfy the boundary conditions at the interface $x=n a$

$$
\psi(n a+0)=\psi(n a-0)
$$

and

$$
\Delta \psi^{\prime}(n a)=\left[\frac{(-1)^{n}}{2} \tilde{g} B \sigma_{z}+i \Delta \alpha(n a) \sigma_{y}+i \Delta \beta(n a) \sigma_{x}\right] \psi(n a),
$$

where $\tilde{g}=g^{*} m^{*} / m_{0}, \quad \Delta \psi^{\prime}(n a)=\psi^{\prime}(n a+0)-\psi^{\prime}(n a-0)$ and $\Delta \alpha(n a)=\alpha(n a+0)-\alpha(n a-0), \Delta \beta(n a)=\beta(n a+0)-\beta(n a-0)$. It is noted that $\Delta \alpha(n a)$ and $\Delta \beta(n a)$ is nonzero only at the ends of the 2DEG. The system can then be regarded as a MKP model except the ends of the 2DEG.

Now let us consider a $N$ periods (i.e., region $[0,(2 N-1) a]$ in Fig. 1) MKP superlattice. For $x<0$, the wave function is given as 


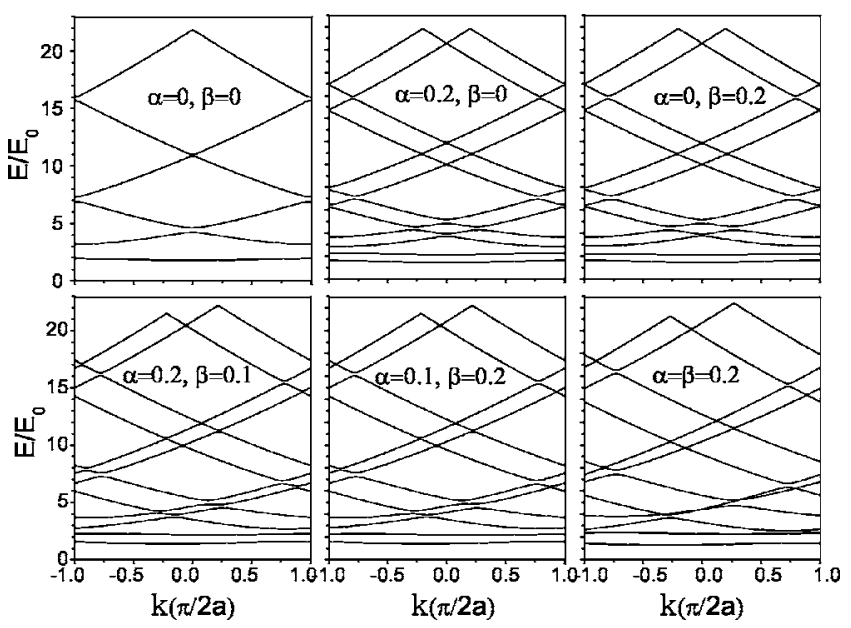

FIG. 2. Energy vs $k$ dispersion curves for various values of $\alpha$ and $\beta$, where $k_{y}=2, a=1.5$, and $B=1$. Only the lowest bands are shown.

$$
\psi(x)=\left(\begin{array}{c}
a_{l} e^{i k_{0} x}+b_{l} e^{-i k_{0} x} \\
c_{l} e^{i k_{0} x}+d_{l} e^{-i k_{0} x}
\end{array}\right)
$$

and for $x>(2 N-1) a$ the solution can be written as

$$
\psi(x)=\left(\begin{array}{c}
a_{r} e^{i k_{0} x} \\
c_{r} e^{i k_{0} x}
\end{array}\right),
$$

where $k_{0}=\sqrt{2 E-\left(k_{y}+A_{2}\right)^{2}}$ (where $A_{1}=B / 2$, and $\left.A_{2}=-B / 2\right)$.

While in the scattering region, each period consists of two segments with different values of vector potentials $A_{1}$ and $A_{2}$. The wave function of each segment can be written according to Eq. (5). Using the boundary conditions (6) and (7), we can calculate the transmission probability through $S$-matrix formalism. ${ }^{20} \mathrm{We}$ choose the incident electron from the left side with a spin-up eigenstate of $\sigma_{z}$. Hence, we have $a_{l}=1$ and $c_{l}=0$ on the left side of finite 2DEG. After electron passing through the scattering region, the electron would be in the superposition state of spin-up and spin-down. The corresponding transmission probabilities are given by

$$
\mathcal{T}_{\uparrow \uparrow}\left(E, k_{y}\right)=\frac{\left|a_{r}\right|^{2}}{\left|a_{l}\right|^{2}}=\left|a_{r}\right|^{2}
$$

and

$$
\mathcal{T}_{\uparrow \downarrow}\left(E, k_{y}\right)=\frac{\left|c_{r}\right|^{2}}{\left|a_{l}\right|^{2}}=\left|c_{r}\right|^{2} .
$$

The total transmission probability is the sum $\mathcal{T}=\mathcal{T}_{\uparrow \uparrow}+\mathcal{T}_{\uparrow \downarrow}$. Similarly we define the transmission probability for spin-up and spin-down electrons $\mathcal{T}_{\downarrow \uparrow}$ and $\mathcal{T}_{\downarrow \downarrow}$ if the incident electron is in the state of spin-down.

\section{BAND STRUCTURE AND TRANSMISSION}

In Fig. 2, we show the electron band structures for various values of $\alpha$ and $\beta$. In the absence of spin-orbit interaction (i.e., $\alpha=\beta=0$ ), the band structure has the usual appearance of energy minibands separated by forbidden gaps. The

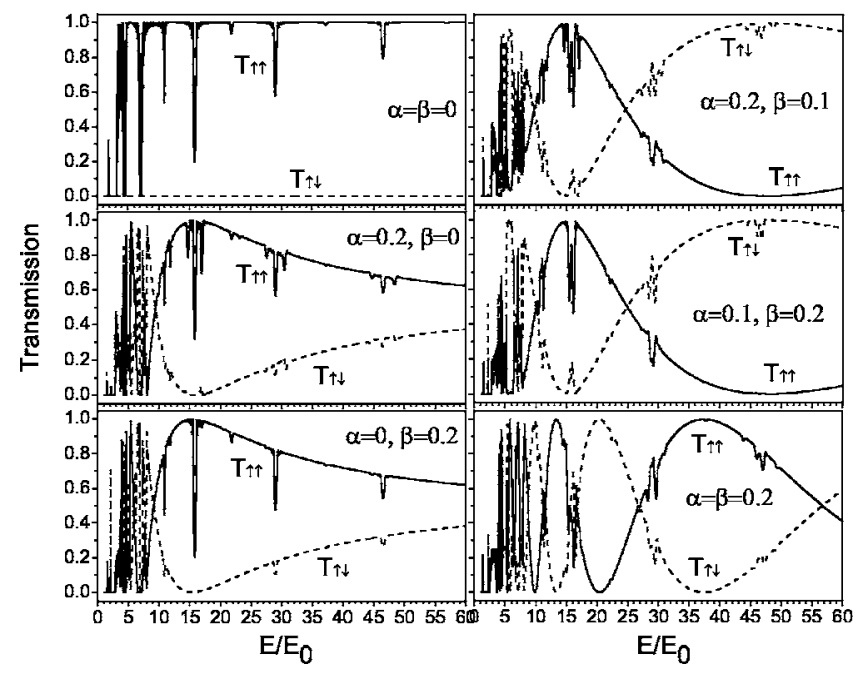

FIG. 3. The transmission probability of spin-up $\left(\mathcal{T}_{\uparrow \uparrow}\right)$ and spindown $\left(\mathcal{T}_{\uparrow \downarrow}\right)$ electrons with fixed incident spin state (spin-up) as a function of incident energy when $\alpha$ and $\beta$ take various values, where $N=20, k_{y}=2, a=1.5, B=1$.

widths of bands and the gaps between them depend on $k_{y}$. It is totally different from those in the EKP superlattice. The dependence of the band structure on $k_{y}$ can be found in Refs. 6 and 7 in detail. For $\alpha=\beta=0$, the degeneracy of spin-up electrons and spin-down electrons is not resolved by the Zeeman term. This is not surprising because $B_{z}(x)$ is antisymmetric so that any differential effect on the up/down spin caused by the positive magnetic field is compensated by the opposite effect induced by the negative magnetic field. ${ }^{21}$

In the presence of spin-orbit interaction, the spin degeneracy is lifted. The band splits into two branches. When only Rashba or Dresselhaus term is considered $(\alpha=0.2, \beta=0$ or $\alpha=0, \beta=0.2$ ), the symmetry between $k_{x}$ and $-k_{x}$ of the band structure remains. But when both $\alpha$ and $\beta$ are nonzero, this symmetry is broken. In addition, when $\alpha$ and $\beta$ exchange values, the band structure keep the same if the Zeeman term is not considered. All these characters discussed above are similar to that of a free $2 \mathrm{DEG} .^{22}$ For the present system with finite bandwidths and gaps, each band for $\alpha=\beta=0$ splits into two spin-subbands for finite $\alpha$ or $\beta$ or the both. As a result, a gap seems to spit into "three gaps" or "four gaps." When only one type of spin-orbit coupling is considered, it appears to be "three gaps" because of the symmetry between $k_{x}$ and $-k_{x}$. When both the two coupling is considered, it appears to be "four gaps" as shown in the lower three panels of Fig. 2.

The energy dependent transmission coefficients are shown in Fig. 3 for various different values of $\alpha$ and $\beta$. Here we include 20 periods $(N=20)$. The total probability $\mathcal{T}$ is insensitive to $N$ when $N$ is larger than 20, while the transmission probability of spin-up or down electrons $\left(\mathcal{T}_{\uparrow \uparrow}\right.$ or $\left.\mathcal{T}_{\uparrow \downarrow}\right)$ oscillate when $N$ increases, because spin-orbit coupling will precess the spin of electron endlessly along with the motion of electron in the $x$ direction. ${ }^{10}$ It is interesting to note that the Rashba term and the Dresslhaus term have the same effect on the spin precession. The energy dependence of $\mathcal{T}_{\uparrow \uparrow}$ ( or $\mathcal{T}_{\uparrow \downarrow}$ ) hardly changes when we interchange the values of $\alpha$ and $\beta$. This behavior can be related to the nearly symmetric role 


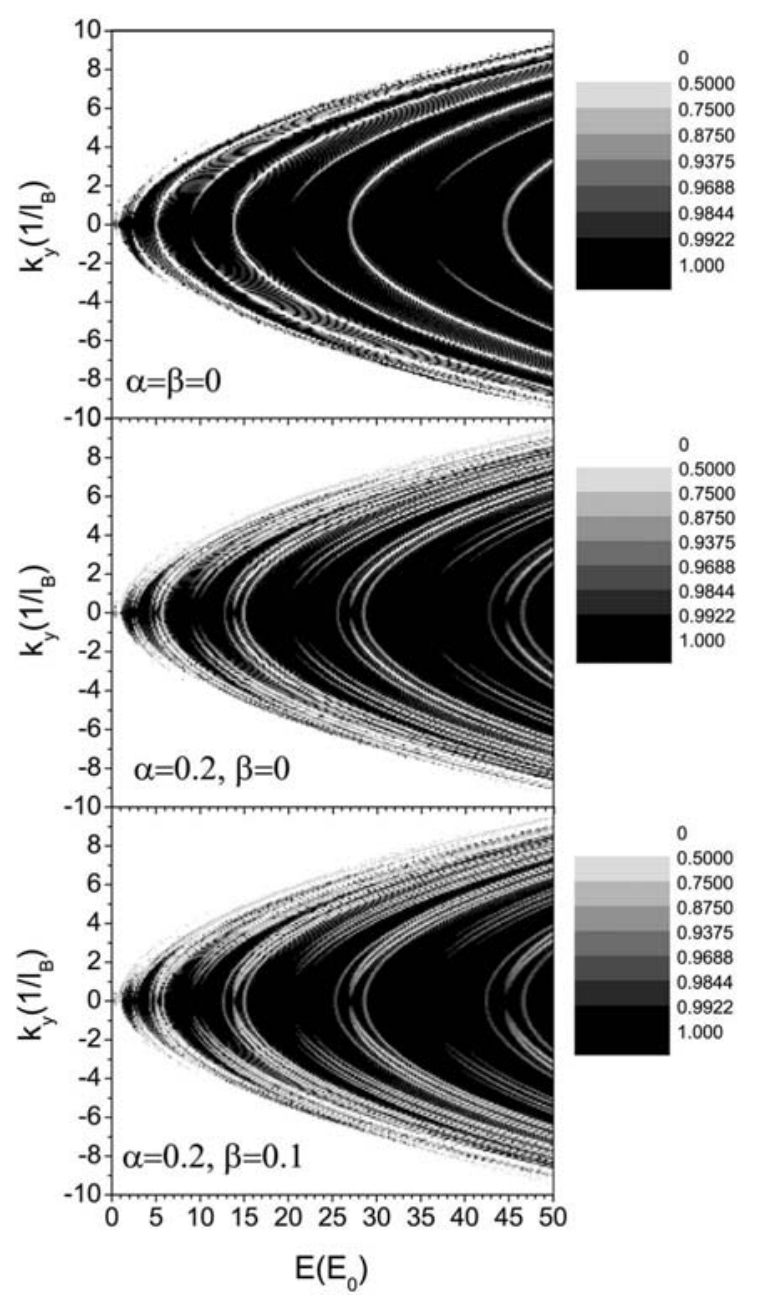

FIG. 4. Contour plot of the total transmission probability $\mathcal{T}$ as a function of energy $E$ and transverse wave vector $k_{y}$ for various values of $\alpha$ and $\beta$. The other parameters are the same as those in Fig. 3.

played by $\alpha$ and $\beta$ in the single particle wave functions of a free electron. It can be shown that the free electron state for

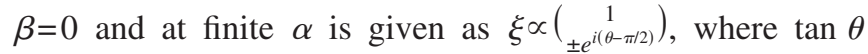
$=k_{y} / k_{x}$. For fixed $k_{y}$ the spin precession is a function of total energy $E$. On the other hand, if $\alpha=0$ and $\beta$ is finite, $\xi$ $\propto\left(\begin{array}{c}1 \\ +e^{-i \theta}\end{array}\right)$. The difference is simply due to the fact the $\sigma_{x}$ couples to $p_{y}$ in the Rashba term and to $p_{x}$ in the Dresslhaus term. Therefore, the behavior of spin precession under the Dresslhaus coupling is the same as that under the Rashba coupling. Moreover, the dips in $\mathcal{T}_{\uparrow \uparrow}(E)$ occur whenever the energy fall in the forbidden gap, i.e., the transmission is blocked. At finite $\alpha$ or $\beta$ or the both, each dip splits into three or four dips, consistent with the change in the band structure.

Figure 4 gives a contour plot of the dependence of the total transmission probability on the energy $E$ and the transverse wave vector $k_{y}$. The different gray scale from white to black corresponds to different values from 0 to 1 . Panel (a) shows the total transmission probability $\mathcal{T}$ for $\alpha=\beta=0$. The white slots are the dips in the transmission probability which corresponds the forbidden gaps in the band structures. We

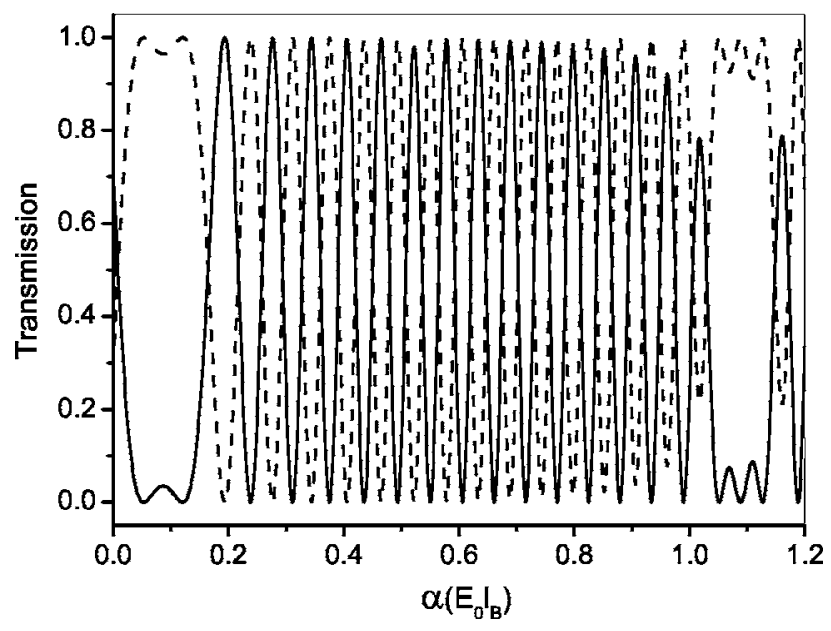

FIG. 5. The transmission probability of spin-up $\left(\mathcal{T}_{\uparrow \uparrow}\right)$ and spindown $\left(\mathcal{T}_{\uparrow \downarrow}\right)$ electrons as a function of Rashba strength $\alpha$ with fixed Dresselhaus coupling strength $\beta=0.2$ and fixed incident Fermi energy $E_{F}=41.2$, and the other parameters are the same as those in Fig. 3.

can see the clear manifestation of the symmetry between $k_{y}$ and $-k_{y}$ and the split of bands when spin-orbit coupling is considered. For $\alpha=0, \beta \neq 0$ and $\alpha \neq 0, \beta=0$, each white slot split into three, shown in panel (b). For $\alpha \neq 0, \beta \neq 0$, each white slot split into four, shown in panel (c).

For an electron with spin up entering the region with finite $\alpha$ and $\beta$, both $\mathcal{T}_{\uparrow \uparrow}$ and $\mathcal{T}_{\uparrow \downarrow}$ oscillate with $\alpha$ and $\beta$ in the form of sinusoidal function. This is common for a transmission probability in any two-level systems. Such oscillatory transmission coefficient is shown in Fig. 5, where $\alpha$ is increased and $\beta$ is fixed to 0.2 .

When the incident spin state is fixed to spin down, we found $\mathcal{T}_{\downarrow \uparrow}$ to be close to $\mathcal{T}_{\uparrow \downarrow}$ and $\mathcal{T}_{\downarrow \downarrow}$ close to $\mathcal{T}_{\uparrow \uparrow}$. The reason is that spin-orbit coupling mostly precesses the spin of electron and does not cause spin filtering directly.

\section{CONDUCTANCE AND COMPARING WITH A REAL SUPERLATTICE}

The conductance of the system can be calculated in the ballistic regime as the average electron flow over half the Fermi surface. ${ }^{16}$ For an incident electron in the spin-up state, the conductance of spin-up $\left(G_{\uparrow \uparrow}\right)$ and spin-down $\left(G_{\uparrow \downarrow}\right)$ of the system are given as

$$
G_{\uparrow \uparrow(\uparrow \downarrow)}=G_{0} \int_{-\pi / 2}^{\pi / 2} \mathcal{T}_{\uparrow \uparrow(\uparrow \downarrow)}\left(E, \sqrt{2 E_{F}} \sin \theta\right) \cos \theta d \theta,
$$

where $\theta$ is the angle of incident relative to the $x$ direction, $G_{0}=e^{2} m^{*} v_{F} L_{y} / h^{2}$, where $L_{y}$ is the length of the structure in the $y$ direction and $v_{F}$ is the Fermi velocity. And the total conductance of spin-up incident electrons is $G=G_{\uparrow \uparrow}+G_{\uparrow \downarrow}$.

Figure 6 shows the conductances as functions of energy at different magnetic fields. First, the conductance curves retain some band structure features such as the dips and oscillations. The sizes of dips and oscillations increase with the magnetic field, because the barrier height of the effective 


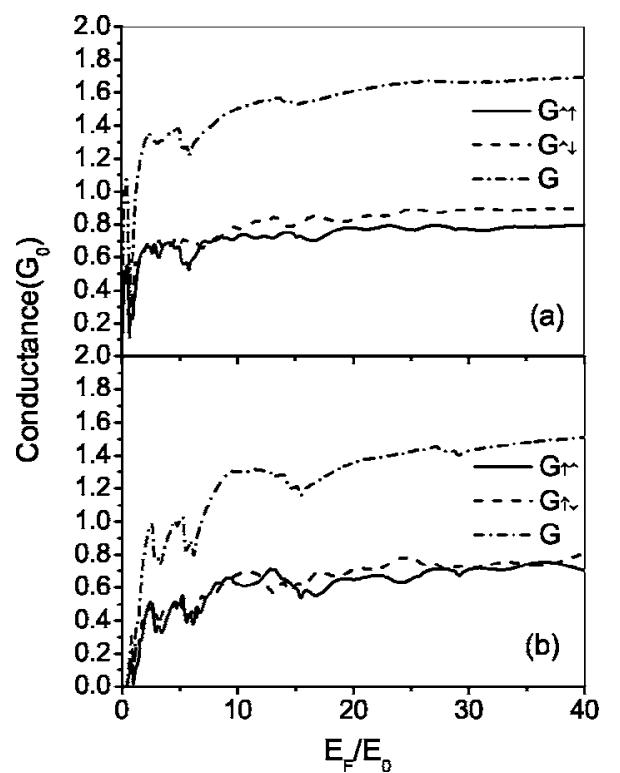

FIG. 6. The conductance $G_{\uparrow \uparrow}$ and $G_{\uparrow \downarrow}$ and the total conductance $G=G_{\uparrow \uparrow}+G_{\uparrow \downarrow}$ as functions of Fermi energy, for different magnetic field: (a) $B=1$, (b) $B=2$. Here $\alpha=0.2, \beta=0.1$ and $N=20, a=1.5$.

potential increases with the magnetic field. Second, the conductances of spin-up and spin-down keep an almost stable rate, which means a stable outgoing spin state.

If we average the barrier height of a MKP superlattice over $k_{y},\left\langle U\left(B, k_{y}\right)\right\rangle=V_{0}=B^{2} / 2$, we can regard the MKP superlattice as an equivalent EKP superlattice with a tunable barrier height controlled by the magnetic field. We can make a comparison of the conductances of the two systems. Figure 7 shows energy dependencies of conductances for a real superlattice (the EKP model) with corresponding barrier height: panel (a) $V_{0}=0.5$ corresponding to $B=1$ and panel (b) $V_{0}=2$ corresponding to $B=2$, accordingly. The conductance curves have very similar band structure features to the MKP model, but with less oscillations. On the whole, the conductances of the two models are very similar. The advantage of using the MKP superlattice is that the barrier is tunable with the magnetic field.

\section{SPIN FILTERING}

Tan $e$ t al. ${ }^{23}$ have discussed spin procession in the presence of spin-orbit coupling. In this part, we focus on the spin filtering effect caused by MKP potential of ferromagnetic strips, in which the in-plane magnetization along the $x$ direction alternating in sign as shown in Fig. 1(a). In our system, if the number of strips in the scattering region is even, the number of $\delta$-function-like magnetic fields pointing to $+z$ will be equal to the number of those pointing to $-z$. In this case, the magnetic field is antisymmetric and causes no spin filter effect. $^{24,25}$ If the number of ferromagnetic strips is odd, the number of $\delta$-function-like magnetic fields pointing to $z$ will be one more or less than the number of those pointing to $-z$. In this case, the magnetic field is symmetric. For the symmetric case, Guo et al. ${ }^{26}$ have shown that symmetric delta barriers can induce a small net polarization with the help of

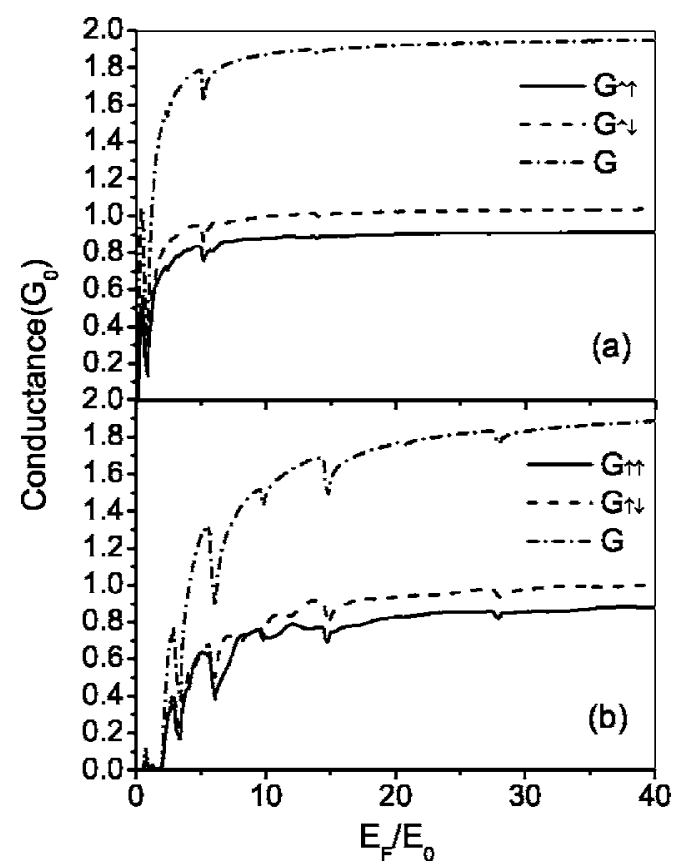

FIG. 7. The conductance $G_{\uparrow \uparrow}$ and $G_{\uparrow \downarrow}$ and the total conductance $G=G_{\uparrow \uparrow}+G_{\uparrow \downarrow}$ as functions of Fermi energy, for a real superlattice with corresponding barrier height: (a) $V_{0}=0.5$, (b) $V_{0}=2$. The other parameters are the same as those in Fig. 6.

electric barrier and this effect was extended to periodic structure to obtain a large polarization. ${ }^{27}$ Our system differs from the previous ones by the boundary of the scattering region. Because there is only one magnetic strip provided in the magnetic field at the edge of the scattering region, the magnetic field of the left boundary and the right boundary ( $x$ $=0$ and $x=2 N a$ ) are only half of the magnetic field in the middle. After taking this detail into account, the symmetric magnetic field case causes a big spin filtering effect without any electric barrier. Figure 8 illustrates the symmetric magnetic field case of our system when the boundary effect is considered.

In the absence of spin flipping mechanism, we can simply define $G_{\uparrow}$ as the conductance of spin-up electrons, $G_{\downarrow}$ the conductance of spin-down electrons, and the total conductance $G=G_{\uparrow}+G_{\downarrow}$. The conductance spin polarization is characterized by the fractional difference between the spin-up and spin-down conductances

$$
P=\frac{G_{\uparrow}-G_{\downarrow}}{G_{\uparrow}+G_{\downarrow}} .
$$

Figure 9 shows the conductance and its spin polarization due to a symmetric magnetic field and the boundary effect for $N=20$. We can see that there are dips in $G_{\uparrow}$ and $G_{\downarrow}$ curves, which embody the band structure of the system. Though the polarization seems approaching to $100 \%$ when Fermi energy values around 0.2 , the conductance is nearly zero. The biggest polarization achievable is around $60 \%$, with the total conductance near 0.05 . The polarization curve is not smooth due to the band structure. 

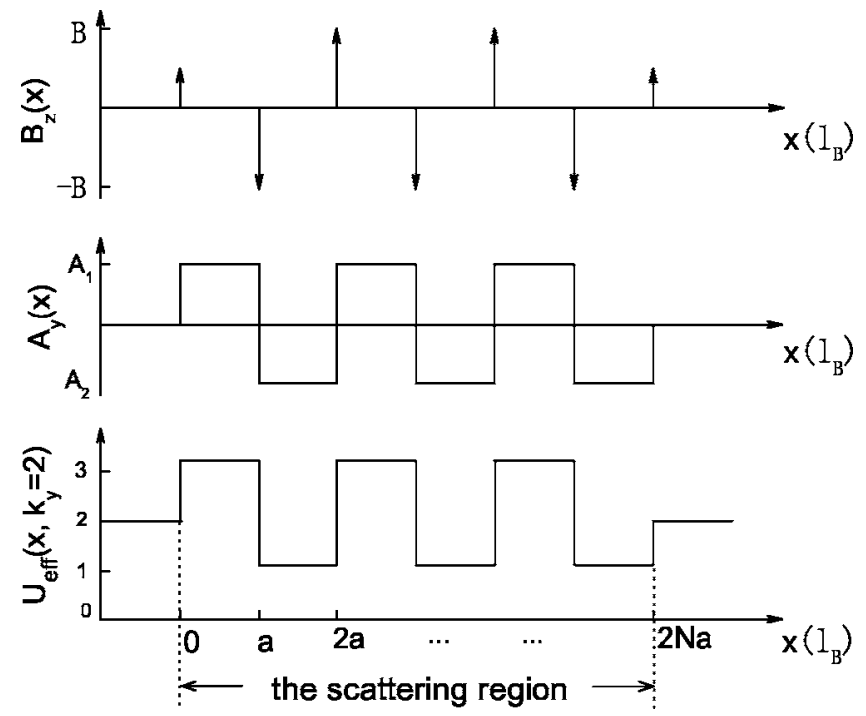

FIG. 8. Schematic illustration of the symmetric magnetic field case of our system when the boundary effect is considered: the model magnetic field, the vector potential, and the effective potential.

Figure 10 shows the conductance and the polarization in a single period structure. The peak of the spin-up conductance is well separated from that of the spin-down conductance, a consequence of the spin dependent resonant tunneling. ${ }^{25}$ The polarization reaches $55 \%$ and the corresponding total conductance is almost 0.1 . The polarization is smooth and stable when energy increases. The energy region with good polarization is wider. This indicates that a single-barrier system is a good spin filter.

Figure 11 gives a contour plot of the conductance spin polarization as a function of the length of half period $a$ and Fermi energy, where $B=1.2, N=1$. For different length of half period $a$, the maximums of polarization as a function of incident energy all occurs at energy around $E=0.182$. It is noteworthy that when $a$ exceeds 11 , the maxima of polarization will exceed $80 \%$. However, the bigger polarization is at

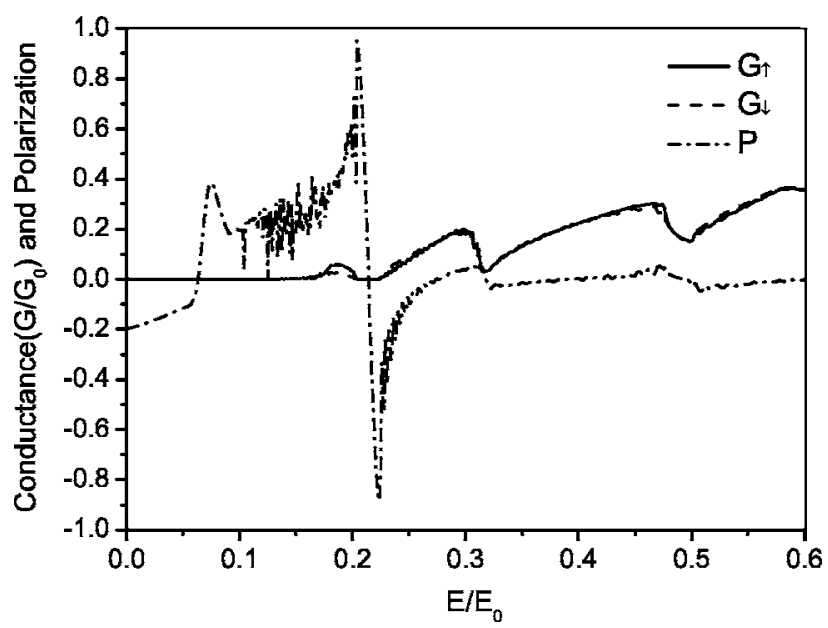

FIG. 9. Conductance of spin-up and spin-down and conductance spin polarization as functions of Fermi energy, where $B=1.2, a=6$, $N=20$.

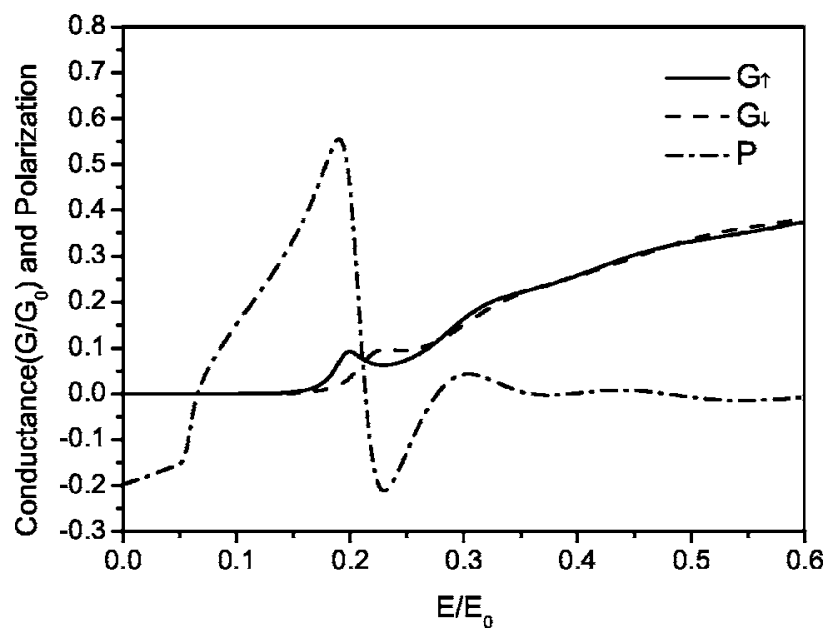

FIG. 10. Conductance of spin-up and spin-down and conductance spin polarization as functions of Fermi energy, where $B=1.2$, $a=6, N=1$.

the cost of the smaller conductance. Figure 12 shows the conductance spin polarization and the total conductance as functions of $a$ with fixed Fermi energy $E=0.182$. We can see when $a$ exceeds 16 , the total conductance reduces to nearly zero.

Figure 13 shows a magnetic field dependence of the conductance spin polarization with Fermi energy $E=0.18,0.20$, and 0.22 . The curves indicate that the magnetic field can be used to tune the conductance spin polarization from its minimum to its maximum. We found the maximum of polarization occurs at different magnetic field values for different incident Fermi energy. The maximum conductance spin polarization at a given Fermi energy can be achieved by adjusting the strength and period of the magnetic modulation.

\section{CONCLUDING REMARKS}

Our study of ballistic transport of 2DEG system through the MKP superlattice in the presence of both Rashba and Dresselhaus spin-orbit coupling has given new insight into

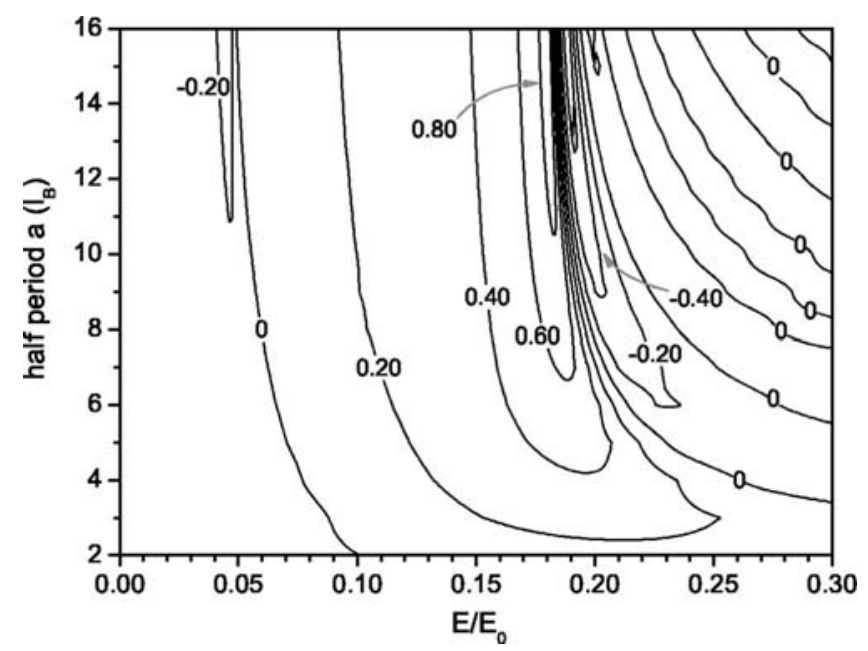

FIG. 11. Contour plot of the conductance spin polarization as a function of the half period $a$ and Fermi energy, where $B=1.2, N$ $=1$. 


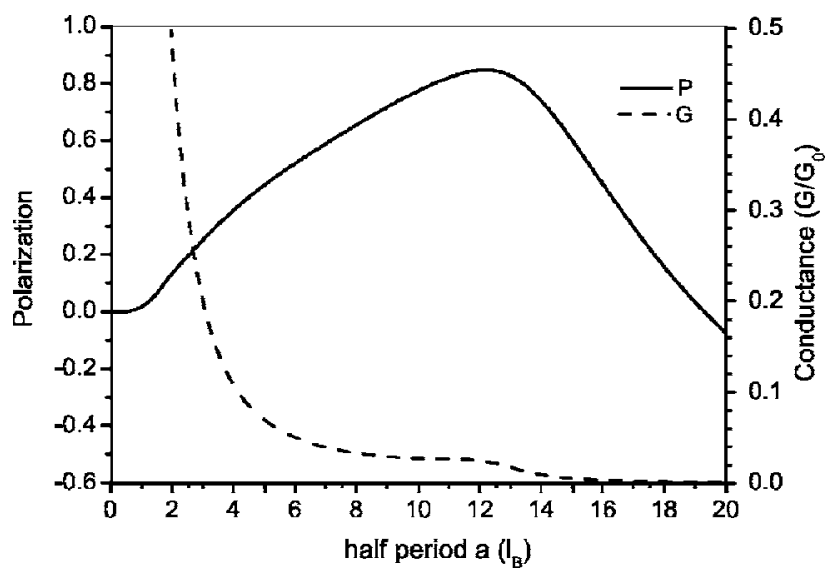

FIG. 12. The conductance spin polarization and the total conductance as functions of the length of half period $a$ with fixed Fermi energy $E=0.182$, where $B=1.2, N=1$.

the interplay between superlattice periodic potential and spin-orbit coupling. The MKP superlattice potential leads to the band gaps which is doubly degenerate. The spin-orbit coupling has lifted the degeneracy and resulted in the electron spin precession. The spin-orbit coupling is also responsible for a series of minima in the transmission probability. When the boundary effect is considered, the system shows a

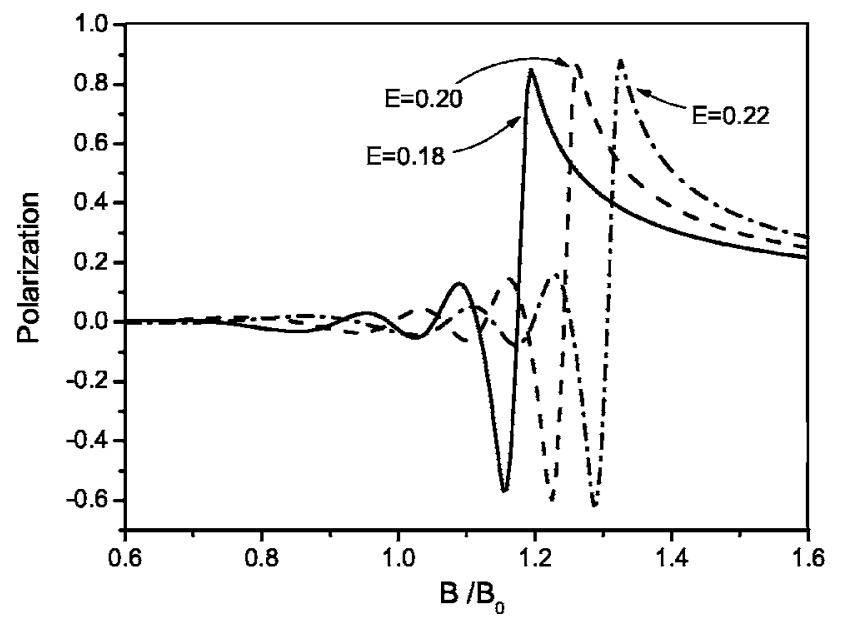

FIG. 13. The conductance spin polarization as function of the magnetic field $B$ with Fermi energy $E=0.18$ (solid curve), $E=0.20$ (dotted curve), and $E=0.22$ (dashed curve), where $a=12, N=1$.

big conductance spin polarization and can be a good candidate for spin filters.

\section{ACKNOWLEDGMENTS}

This work was supported in part by RFDP, NNSFC Grant Nos. 10274069, 10474002, and 90303014, and the Australian Research Council.
${ }^{1}$ D. Weiss, K. von Klitzing, K. Ploog, and G. Weimann, Europhys. Lett. 8, 179 (1989); R. R. Gerhardts, D. Weiss, and K. v. Klitzing, Phys. Rev. Lett. 62, 1173 (1989); R. W. Winkler, J. P. Kotthaus, and K. Ploog, ibid. 62, 1177 (1989); C. Zhang and R. R. Gerhardts, Phys. Rev. B 41, 12850 (1990).

${ }^{2}$ J. E. Muller, Phys. Rev. Lett. 68, 385 (1992); F. M. Peeters and A. Matulis, Phys. Rev. B 48, 15166 (1993); S. M. Stewart and C. Zhang, J. Phys.: Condens. Matter 10, 5545 (1998).

${ }^{3}$ A. Nogaret, S. Carlton, B. L. Gallagher, P. C. Main, M. Henini, R. Wirtz, R. Newbury, M. A. Howson, and S. P. Beaumont, Phys. Rev. B 55, R16037 (1997).

${ }^{4}$ H. A. Carmona et al., Phys. Rev. Lett. 74, 3009 (1995).

${ }^{5}$ M. Kato, A. Endo, S. Katsumoto, and Y. Iye, Phys. Rev. B 58, 4876 (1998).

${ }^{6}$ I. S. Ibrahim and F. M. Peeters, Am. J. Phys. 63, 171 (1995).

${ }^{7}$ I. S. Ibrahim and F. M. Peeters, Phys. Rev. B 52, 17321 (1995).

${ }^{8}$ V. Kubrak, F. Rahman, B. L. Gallagher, P. C. Main, M. Henini, C. H. Marrows, and M. A. Howson, Appl. Phys. Lett. 74, 2507 (1999).

${ }^{9}$ C. M. Hu, J. Nitta, A. Jensen, J. B. Hansen, and H. Takayanagi, Phys. Rev. B 63, 125333 (2001).

${ }^{10}$ S. Datta and B. Das, Appl. Phys. Lett. 56, 665 (1990).

${ }^{11}$ J. S. Moon et al., Appl. Phys. Lett. 85, 678 (2004).

${ }^{12}$ Hong Chen, J. J. Heremans, J. A. Peters, and A. O. Govorov, Appl. Phys. Lett. 86, 032113 (2005).

${ }^{13}$ M. L. Leadbeater et al., J. Phys.: Condens. Matter 7, L307
(1995).

${ }^{14}$ E. I. Rashba, Fiz. Tverd. Tela (Leningrad) 2, 1224 (1960) [Sov. Phys. Solid State 2, 1109 (1960)]; Y. A. Bychkov and E. I. Rashba, J. Phys. C 17, 6039 (1984).

${ }^{15}$ G. Dresselhaus, Phys. Rev. 100, 580 (1955).

${ }^{16}$ G. Papp and F. M. Peeters, Appl. Phys. Lett. 78, 2184 (2001).

${ }^{17}$ Junsaku Nitta, Tatsushi Akazaki, Hideaki Takayanagi, and T. Enoki, Phys. Rev. Lett. 78, 1335 (1997).

${ }^{18}$ P. D. Ye, D. Weiss, R. R. Gerhardts, and H. Nickel, J. Appl. Phys. 81, 5444 (1997).

${ }^{19}$ A. Matulis, F. M. Peeters, and P. Vasilopoulos, Phys. Rev. Lett. 72, 1518 (1994).

${ }^{20} \mathrm{~S}$. Datta, in Electronic Transport in Mesoscopic Systems (Cambridge University Press, Cambridge, 2002).

${ }^{21}$ Y. Jiang and M. B. A. Jail, J. Phys.: Condens. Matter 15, L31 (2003).

${ }^{22}$ Shun-Qing Shen, Phys. Rev. B 70, 081311(R) (2004).

${ }^{23}$ S. G. Tan et al. (unpublished); J. Appl. Phys. 97, 10 D506 (2005); 97, 10A716 (2005).

${ }^{24}$ H. Z. Xu and Y. Okada, Appl. Phys. Lett. 79, 3119 (2001); Y. Jiang, M. B. A. Jalil, and T. S. Low, ibid. 80, 1673 (2002).

${ }^{25}$ Feng Zhai, H. Q. Xu, and Y. Guo, Phys. Rev. B 70, 085308 (2004).

${ }^{26}$ Y. Guo, J. H. Qin, X. Y. Chen, and B. L. Gu, Semicond. Sci. Technol. 18, 297 (2003).

${ }^{27}$ M. B. A. Jalil et al., J. Appl. Phys. 95, 7321 (2004). 